\title{
Simulation of Local Dissipation Phenomena in the REBCO Insert of the 25-T CSM Magnet: Understanding and Preventing Destructive Thermal Runaway
}

\author{
Arnaud Badel ${ }^{\circledR}$, Blandine Rozier ${ }^{\circledR}$, Kohki Takahashi ${ }^{\circledR}$, and Satoshi Awaji ${ }^{\circledR}$
}

\begin{abstract}
We recently developed an electrothermal model to study the occurrence and evolution of dissipative zones in a RareEarth $\mathrm{BaCuO}$ (REBCO) high-temperature superconductor (HTS) coils, considering the local critical current $\left(I_{c}\right)$ inhomogeneity with a centimeter-scale resolution. In this study, we present an application of this model, implemented in Comsol Multiphysics, to the REBCO insert of the 25-T CSM magnet developed in the High Field Laboratory for Superconducting Material of Tohoku University. The destructive thermal runaway event or "quench" observed on that coil will be introduced, followed by the modeling approach that was used to model it. We could simulate the coil behavior with a single 2 -cm-long defect and verify that a more sensitive detection system in the millivolt range could be used to safely protect such magnets even from such a local defect. In the case of a more usual inhomogeneity distribution of $I_{\mathrm{c}}$, the temperature rise is more homogenous and safe protection can be reached even at higher detection levels.
\end{abstract}

Index Terms-HTS magnets, quench propagation, very high field and nmr magnets.

\section{CONTEXT And Motivation}

$\mathbf{R}$ ARE-EARTH BaCuO (REBCO) High Temperature Superconductors (HTS) are attractive for very high field inserts due to their large engineering current densities under high magnetic fields when operating close to $4.2 \mathrm{~K}$, combined with their good mechanical strength. That is the reason why it was the first choice for designing the HTS insert of the $25 \mathrm{~T}$ Cryogen-free Superconducting Magnet (CSM) developed at the High Field Laboratory for Superconducting Material [1]. Specific developments were conducted on REBCO tapes produced by Fujikura for that project. They could typically carry around $400 \mathrm{~A} / \mathrm{cm}$-width under $20 \mathrm{~T}$ transverse field at $20 \mathrm{~K}$ and sustain more than $780 \mathrm{MPa}$ without degradation [2]. The initial design

A. Badel was with CNRS and Université Grenoble Alpes, Grenoble 38042, France. He is now with Tohoku University, Sendai 980-8577, Japan (e-mail: arnaud.badel@imr.tohoku.ac.jp).

B. Rozier is with the Université Grenoble Alpes, Grenoble 38042, France (e-mail: blandine.rozier@g2elab.grenoble.inp.fr).

K. Takahashi and S. Awaji are with Tohoku University, Sendai 980-8577, Japan (e-mail: kohki@imr.tohoku.ac.jp; awaji@imr.tohoku.ac.jp).
TABLE I

25 T CSM REBCO INSERT CHARACTERISTICS

\begin{tabular}{lll}
\hline \hline Current & $\mathrm{A}$ & 144 \\
Average current density & A/mm2 & 129.8 \\
\hline Inner radius & $\mathrm{mm}$ & 52 \\
\hline Outer radius & $\mathrm{mm}$ & 131.42 \\
\hline Height & $\mathrm{mm}$ & 336 \\
\hline Space current density & $\mathrm{A} / \mathrm{mm}^{2}$ & 129.8 \\
\hline Pancake number & - & 56 \\
\hline Turns / pancake & - & 435 \\
\hline B axial max & $\mathrm{T}$ & 25.6 \\
\hline Br & $\mathrm{T}$ & 4.66 \\
\hline B axial (standalone) & $\mathrm{T}$ & 11.5 \\
\hline Width of conductor & $\mathrm{mm}$ & 5.00 \\
\hline Thickness of conductor & $\mathrm{mm}$ & 0.13 \\
\hline Thickness of layer insulation & $\mathrm{mm}$ & 0.055 \\
\hline Hoop Stress BJR & $\mathrm{MPa}$ & 407
\end{tabular}

consisted in a $14 \mathrm{~T}$ Low Temperature Superconducting (LTS) outsert and an 11.5 T REBCO insert cooled down independently, respectively to around $5 \mathrm{~K}$ and $7 \mathrm{~K}$, using a conduction cooling system detailed in [3]. The characteristics of the REBCO insert are summarized in Table I.

Commercial REBCO tapes display performance inhomogeneity along their lengths, with mm-scale variations of the local critical current measured continuously at $77 \mathrm{~K}$ in self-field conditions. Combined with their very good thermal stability, it leads to a high risk of damaging hot spots when operating close to the estimated critical current, as in that case the critical current may be overstepped locally on the weaker zones, leading to local damaging hotspots.

In the case of the $25 \mathrm{~T}$ CSM REBCO insert, the choice was made to use a very large (more than 50\%) current margin [4] in order to limit the risk of such local critical current overstepping. In principle, the magnet could operate as high as $20 \mathrm{~K}$. Moreover, 



Fig. 1. Top, evolution of detection bridge voltage (Balance V) and coil current up to the thermal runaway event (from [7]). Bottom, current evolution during discharge (interpolated from experiment data).

all the REBCO tape lengths used in the magnet were controlled against unusual local defects using continuous measurement under $77 \mathrm{~K}$ self-field [5]. They typically showed maximum local variation of $\pm 7.5 \%$ around the Ic $77 \mathrm{Ks.f}$ mean value. Then, each of the pancakes were tested individually at $77 \mathrm{~K}$ to ascertain that the conductor was not damaged during the process [6]. In operation, both the outsert and the insert were protected by classical bridge detection system triggering discharges in dump resistors. The bridge threshold voltage in the case of the REBCO insert was set to $1-2 \mathrm{~V}$, with a delay of $0.5 \mathrm{~s}$. That protection system was mostly meant to protect the REBCO insert in case of quench induced by the quench of the outsert. The REBCO insert was tested successfully in standalone, but a destructive thermal runaway occurred during the first ramp up under background field, at $124.6 \mathrm{~A}$ (94\% of the nominal value) [7]. The field reached at that moment $24 \mathrm{~T}$. Later on it was replaced by an $11 \mathrm{~T}$ BSCCO insert [8], with which the $25 \mathrm{~T}$ CSM is now operational [7].

The evolution of voltage and current around the quench event is shown Fig. 1. The objective of this work is to use numerical modelling in order to study the possible origins and the dynamic of that event, and to evaluate how this behaviour would have been improved using a more sensitive detection system. The results of the simulation study will be presented and discussed in part III, after the modeling approach is introduced in part II.

\section{Modelling APPROACH}

REBCO coated conductor are thin tapes with multilayered structure. In the case of Fujikura's tape for the 25 T CSM insert, the tape is $5 \mathrm{~mm}$ wide, with a $2.5 \mu \mathrm{m} \mathrm{REBCO} \mathrm{layer} \mathrm{grown} \mathrm{on} \mathrm{a}$ $75 \mu \mathrm{m}$-thick Hastelloy C276 substrate through a number of thin buffer layers of about $0.5 \mu \mathrm{m}$ in total. It is surrounded by $2 \mu \mathrm{m}$ of Silver and $20 \mu \mathrm{m}$ of Copper (Fig. 2). Both the thermal and electrical properties of REBCO tapes are strongly non-linear.

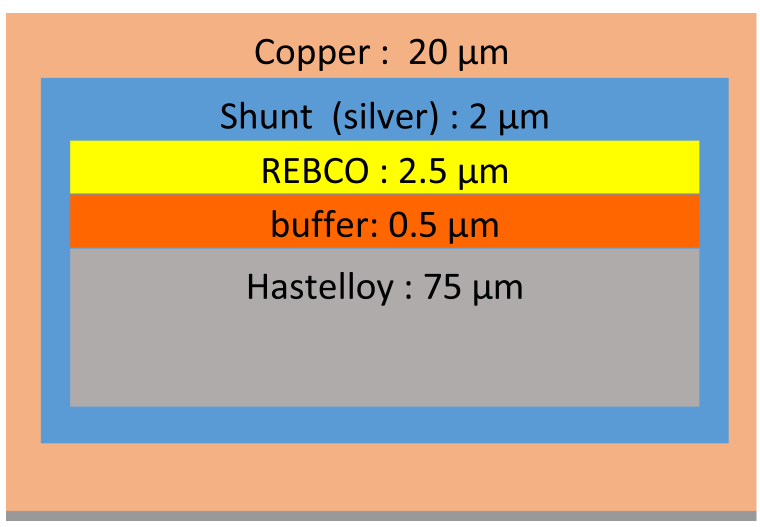

Fig. 2. Cross section of Fujikura tape used in the REBCO insert under study.

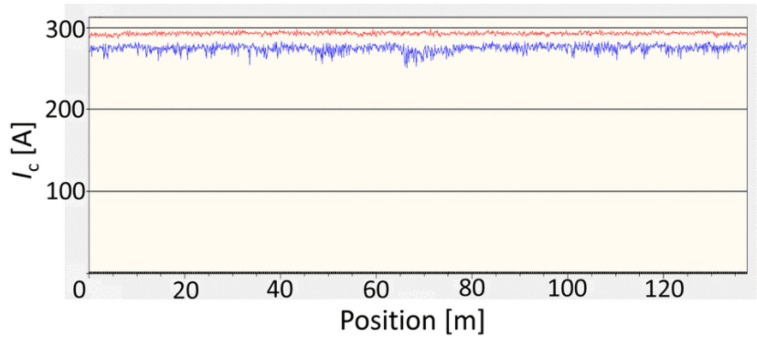

Fig. 3. Typical Ic distribution (lower curve) of $5 \mathrm{~mm}$-wide Fujikura tape used in the project, at $77 \mathrm{~K}$ self field (taken from [2]).

Together with the high aspect ratio of the layers, it would make the solving of the local transient coupled thermal-electric behaviour everywhere in the magnet very time-consuming, if not impossible in the general case. For this reason, we use homogenization as well domain study reduction in our model as will be explained in the next paragraphs. The model is implemented in Comsol Multiphysics as a transient heat transfer problem. The electric model is contained in external functions that are called for each blocks before each time step to evaluate the local heat generation. We will introduce briefly here the model hypothesis approach, the modelling implementation is presented in more details in [9].

\section{A. Electrical Model}

From the electrical point of view, we homogenize the tape at the cross-section level but discretize its length. This is coherent with the available characterization data for the tape critical current that is given at a given position on the length but for the whole tape. Fig. 3 show an example of typical in-length characterization data for Fujikura's tape used in the REBCO insert.

As in our case the magnet is wound with single tape conductor, this results in an equivalent circuit consisting in a string of seriesconnected non-linear variable resistances.

For each elements of the 1D in-length discretization of the electrical problem (called "blocks" in the following) the local critical current must be evaluated in order to compute the equivalent resistance. This local critical current depends from the local tape performances and the operation conditions in terms of field (amplitude and orientation) and temperature. 


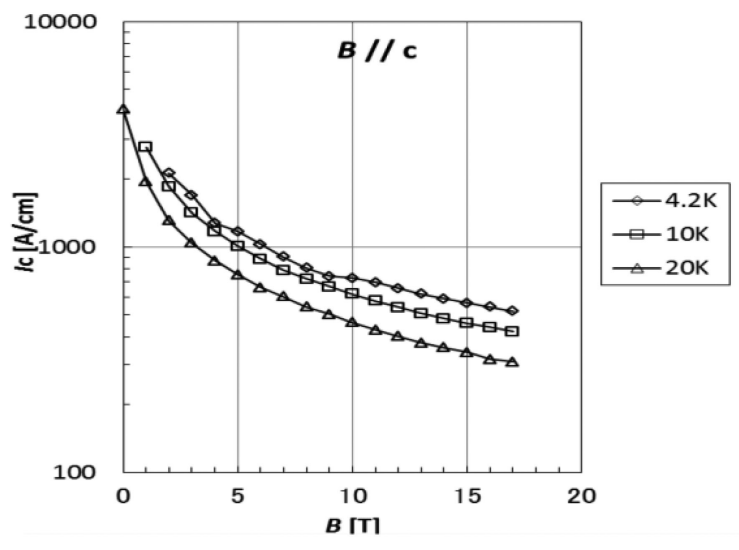

Fig. 4. Variation of Ic per Unit width with Field in Transverse Direction at Various Temperature (from [8]).

We first evaluated a "nominal" distribution of Ic based on short sample characterization, then modulated it by a "tape performance" coefficient varying around unity in a range consistent with the spread observed in continous Ic $77 \mathrm{~K}$ s.f measurement. The exact variation of Ic in a coil cannot be obtained (for existing coils) or foreseen (for future ones). Our aim by modulating the "expected" Ic with distributed defects is simply to give to the model an input Ic distribution that is plausible, and study its influence on the overall behaviour. Fig. 4 display an exemple of Fujikura carcaterization data [10] used as reference values.

Once the critical current for each block is obtained, the equivalent resistance per block is evaluated taking into account the current sharing with the other layers, using iterative methods.

\section{B. Thermal Model Definition}

Considering the pancake strcuture of the winding, we can limit the domain to 2D and study one pancake independently from the others. Knowing from preliminary studies where the weakest Ic is most likely to be found, the thermal model geometry can be defined.

For the numerical modelling of the transient thermal problem, we homogenize the tape thermal properties. The layered structure leads to a strong anisotropic thermal conductivity that must be considered. The co-wound insulation is a $55 \mu \mathrm{m}$ meterthick teflon-coated polyimide tape. It is a distinct layer between each turn in the model, in order to keep the possibility to vary its thickness and to apply thermally resistive interfaces between the turns.

Finally, the 25 T CSM insert pancakes were actually impreganted but the impregantion material was mostly kept out of the winding thanks to the winding tension. We neglect it in our model.

\section{Application to the 25 T CSM RebCo InSERT}

\section{A. Magnetostatic Study and Domain Reduction}

2D axi-symmetric magnetostatic studies are conducted to get the field contributions of both the outsert and the insert for a given combination of insert and outsert current. It is used to to evaluate the "expected" local Jc on every turn of every pancakes
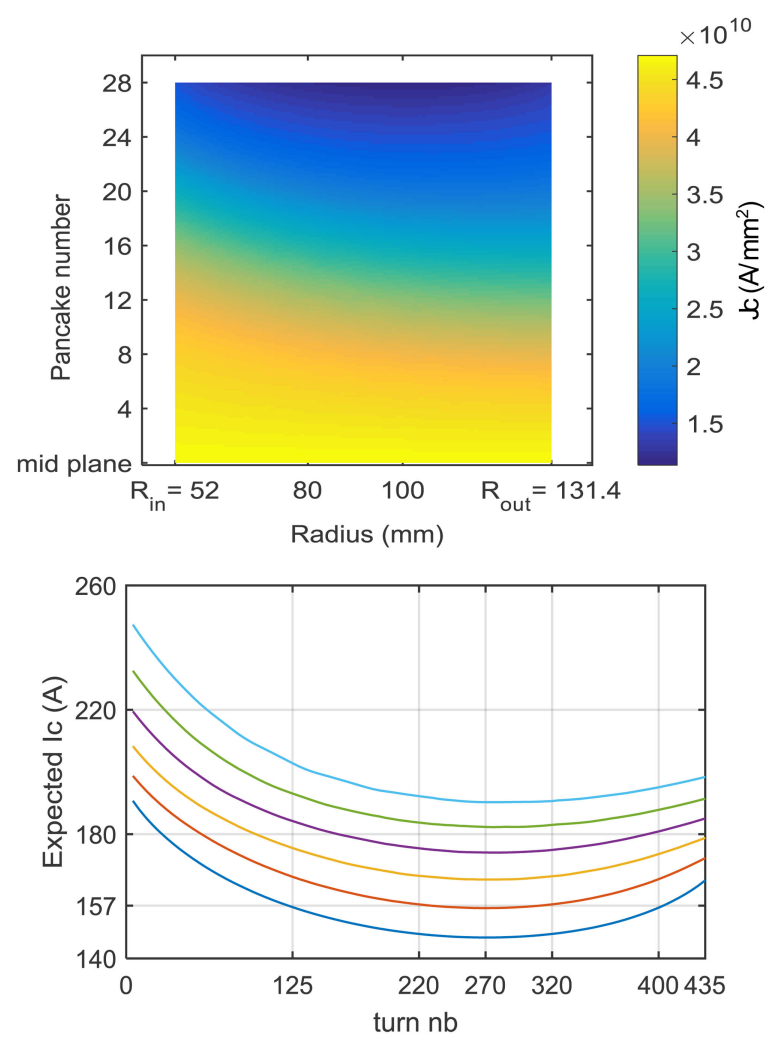

Fig. 5. Top, Jc distribution in the REBCO insert based on magnetostatic study of the 25 T CSM in nominal operation. Bottom, Turn by turn variation of Ic in the top pancakes of the insert under nominal operation. lowest curve is top pancake, second one is $2^{\text {nd }}$ from the top and so on.

(Fig. 5), which are then averaged on the turn width to obtain the local Ic. In the Fujikura tapes used in the REBCO insert, the variation of Ic at $77 \mathrm{~K}$ s.f. was typically below $\pm 7.5 \%$. A study of all the turns in which the expected Ic is at most $7.5 \%$ higher than the lowest expected Ic (i.e., lower than $157 \mathrm{~A}$ ) should include the area in which the risk of dissipation occurrence is maximum.

In our study, it represents most of the top pancake (from turn 125 to 400) and does not concern the others. However, the total tape length to be considered is still more than $190 \mathrm{~m}$, which would generate a model too heavy to handle. In that study we limited ourselves to 100 turns, centered arround the weakest one, that is turn 270. In the eletrical model the conductor length is discretized in about 3000 elements. For each of these elements, which are approximately $2 \mathrm{~cm}$ long, an Ic value was thus set based on the turns expected Ic modulated by a random coefficent between 0.925 and 1.075 .

The initial operating temperature is $6.5 \mathrm{~K}$, obtained from measurement on the real insert. To represent the conduction cooling in the $2 \mathrm{D}$ model, an out-of-plane heat exchange of $2 \mathrm{~W} / \mathrm{m}^{2} . \mathrm{K}$ is considered on both pancake flat surfaces from a cold plate at $6 \mathrm{~K}$. This value is derived from the available cooling power of the system at that temperature.

\section{B. Simulation Procedure}

In the model study the background field is considered already established at nominal value (14 $\mathrm{T}$ in the center). In order 


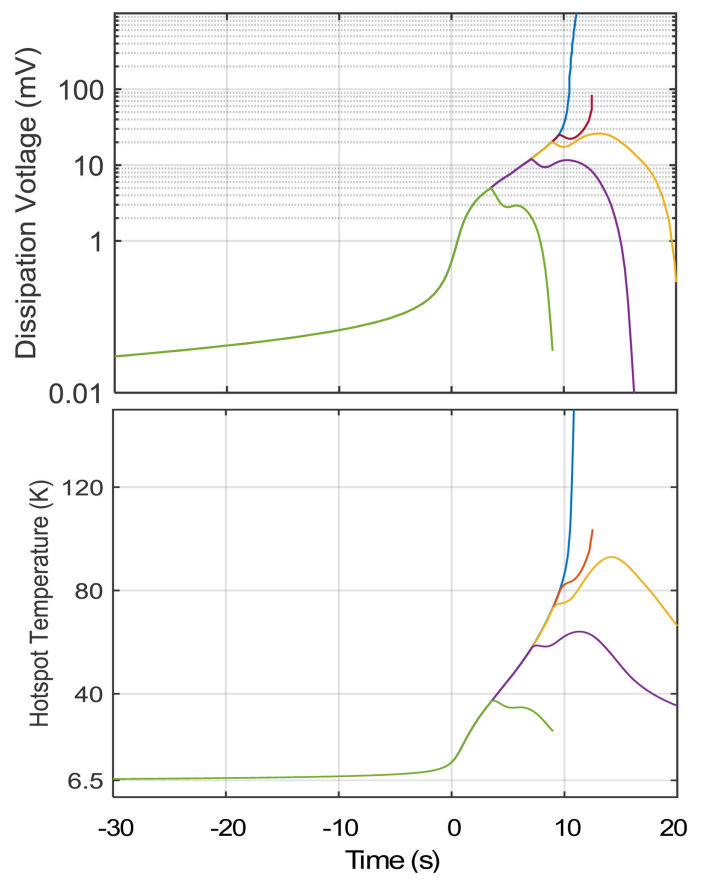

Fig. 6. Dissipating voltage (top) and hotspot temperature (bottom) evolution for various protection discharge thresholds. Origin of time was set arbitrarily.

to shorten the simulation time, the insert current is rised at a constant ramp rate of $0.1 \mathrm{~A} / \mathrm{s}$, while in reality it was closer to $0.017 \mathrm{~A} / \mathrm{s}$. Simulations were conducted on few cases at the two speeds to verify that such ramp rate change had no significant impact. The total voltage across the studied domain is evaluated, and dicharge of the current is initiated after it reaches a given detection threshold. The current discharge profile after the quench protection trigger is known from experiment (see Fig. 1), so the simulation was made with driven current, the preregistered current profile being imposed during the discharge. This way were able to circumvent the limitations of the implementation of our model in Comsol that does not allow easily the coupling of electrical circuits. We are currently developping a more flexible implementation of the model [9] that includes such coupling, but for now it cannot manage a geometry with the dimensions considered here.

\section{Results and Discussion}

The 25 T CSM REBCO insert was damaged during operation at current lower than the nominal, which was already defined with a large margin. The most likely reason was the existance of a sharp local defect. We could reproduce a thermal runway of the magnet at the observed current (around $125 \mathrm{~A}$ ) with a single $2 \mathrm{~cm}$-long defect having only $18.8 \%$ of the expected Ic. The resulting voltage and hotspot temperature evolution are shown Fig. 6 for various detection threshold.

We first used the protection setting used in the experiment, $1 \mathrm{~V}$. In that case we could observe that thermal runaway occurs but does not destroy the coil immediately. However, the model evaluate the hotpost temperature at $600^{\circ} \mathrm{C}$ (destruction) $2 \mathrm{~s}$ only after the discharge is trigered, instead of more than $6 \mathrm{~s}$ in experiment. This may be explained by the fact that our

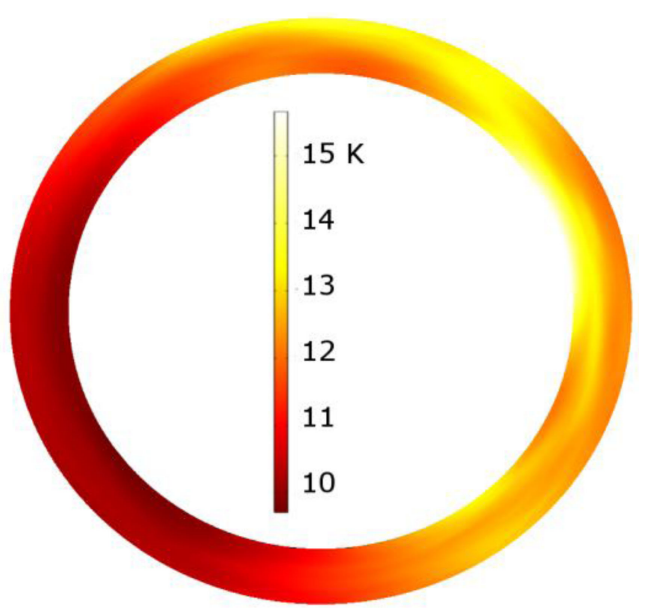

Fig. 7. Temperature distribution at the beginning of the protection discharge for a $20 \mathrm{mV}$ trigger and $\pm 7.5 \%$ distributed conductor inhomogeneity.

model represents only a quarter of a pancake volume as only 100 turns are modelled out of 435 . In case of large heat generation, this limits the spread of the heat diffusion and leads to an overestimation of the peek temperature.

Then we studied the possibilities offered by early detection in such experimental case, varying the threshold value (Fig. 6). The thermal runaway can be prevented even considering the rather slow discharge of the insert and its coupling with the outsert, provided that the detection threshold is lower than $20 \mathrm{mV}$. This value was obtained with no protection delay. A trigger around $12-18 \mathrm{mV}$ would make it possible to wait more than a second (10 s on the graph corresponds to a $1 \mathrm{~A}$ variation before the discharge) to confirm the detection event. In order to minimize fatigue due to local temperature rise, the trigger should be set between 5 and $10 \mathrm{mV}$ so that the temperature is kept below $50 \mathrm{~K}$.

However, these studies are made in the worst possible scenario, that of a single sharp defect. For comparison purpose we then studied the same insert, but with Ic properties homogeneously reduced to $23 \%$ of their expected value, so that the thermal runaway occurs at the same current. The dissipation is more homogenous (Fig. 7). A $20 \mathrm{mV}$ threshold value leads to a maximum temperature of only $17 \mathrm{~K}$.

\section{CONCLUSION}

We recently developped a model to study the occurrence and evolution of dissipative zones in REBCO HTS magnets and the resulting thermal runway phenomenon, taking into account the local inhomogeneity of REBCO tapes performances. This model was used to investigate the destructive thermal runway that occurred in the REBCO insert of the $25 \mathrm{~T}$ CSM magnet developed at the HFLSM of Tohoku University. Although imperfectly, we could reproduce such event and demonstrate that a more sensitive detection system, with threshold value in the $\mathrm{mV}$ range, could have been used to protect such insert effectively from sharp local defects, while detection system in the tens of $\mathrm{mV}$ range would be sufficient in case of a more typical distribution of inhomogeneity. 


\section{REFERENCES}

[1] S. Awaji et al., "New $25 \mathrm{~T}$ cryogen-free superconducting magnet project at Tohoku university," IEEE Trans. Appl. Supercond., vol. 24, no. 3, Jun. 2014, Art. no. 4302005.

[2] S. Fujita et al., "Characteristics of REBCO coated conductors for $25 \mathrm{~T}$ cryogen-free superconducting magnet," IEEE Trans. Appl. Supercond, vol. 25, no. 3, Jun. 2015, Art. no. 8400304.

[3] M. Takahashi et al., "Design and test results of a cryogenic cooling system for a 25-T cryogen-free superconducting magnet," IEEE Trans. Appl. Supercond., vol. 27, no. 4, Jun. 2017, Art. no. 4603805.

[4] H. Miyazaki et al., "Design of a REBCO insert coil for a cryogen-free 25-T superconducting magnet," IEEE Trans. Appl. Supercond., vol. 25, no. 3, Jun. 2015, Art. no. 0500304.

[5] "TAPESTAR-Family: Quality Management for Coated Conductors." [Online]. Available: https://www.theva.com/products/.
[6] S. Awaji, "10 T generation by an epoxy impregnated GdBCO insert coil for the 25 T-cryogen-free superconducting magnet," Supercond. Sci. Technol., vol. 29, no. 5, 2016, Art. no. 055010.

[7] S. Awaji et al., "First performance test of a $25 \mathrm{~T}$ cryogen-free superconducting magnet," Supercond. Sci. Technol., vol. 30, no. 6, 2017, Art. no. 065001 .

[8] S. Hanai, T. Tsuchihashi, S. Ioka, K. Watanabe, S. Awaji, and H. Oguro, "Development of an $11 \mathrm{~T}$ BSCCO insert coil for a $25 \mathrm{~T}$ cryogen-free superconducting magnet," IEEE Trans. Appl. Supercond., vol. 27, no. 4, Jun. 2017, Art. no. 4602406.

[9] A. Badel, B. Rozier, B. Ramdane, G. Meunier, and P. Tixador "Modelling of 'Quench' or the occurrence and propagation of dissipative zones in REBCO HTS coils," Supercond. Sci. Technol., submitted for publication.

[10] S Fujita et al., "In-field critical current property of IBAD/PLD coated conductors," J. Phys.: Conf. Series, vol. 507, no. 2, 2014, Art. no. 022007 . 